\title{
Gyro-resonant electron acceleration at Jupiter
}

\author{
RICHARD B. HORNE ${ }^{1 *}$, RICHARD M. THORNE², SARAH A. GLAUERT ${ }^{1}$, J. DOUGLAS MENIETT| ${ }^{3}$, \\ YURI Y. SHPRITS ${ }^{2}$ AND DONALD A. GURNETT ${ }^{3}$ \\ ${ }^{1}$ British Antarctic Survey, Madingley Road, Cambridge CB3 OET, UK \\ ${ }^{2}$ Department of Atmospheric and Oceanic Sciences, University of California, Los Angeles 90095-1565, USA \\ ${ }^{3}$ Department of Physics and Astronomy, University of lowa, lowa 52242-1479, USA \\ *e-mail: R.Horne@bas.ac.uk
}

Published online: 9 March 2008; doi:10.1038/nphys897

According to existing theory, electrons are accelerated up to ultra-relativistic energies ${ }^{1}$ inside Jupiter's magnetic field by betatron and Fermi processes as a result of radial diffusion towards the planet and conservation of the first two adiabatic invariants ${ }^{2-4}$. Recently, it has been shown that gyro-resonant electron acceleration by whistler-mode waves $^{5,6}$ is a major, if not dominant ${ }^{7}$, process for accelerating electrons inside the Earth's outer radiation zone, and has redefined our concept for producing the Van Allen radiation belts ${ }^{8}$. Here, we present a survey of data from the Galileo spacecraft at Jupiter, which shows that intense whistler-mode waves are observed outside the orbit of the moon Io and, using Fokker-Planck simulations, are strong enough to accelerate electrons to relativistic energies on timescales comparable to that for electron transport. Gyroresonant acceleration is most effective between 6 and 12 jovian radii $\left(\boldsymbol{R}_{\mathrm{j}}\right)$ and provides the missing step in the production of intense synchrotron radiation from Jupiter ${ }^{1,9}$.

At Jupiter, volcanic activity on the moon Io provides a major source of gaseous material ${ }^{10}$ which is ionized by electron impact and solar radiation and forms a torus-like structure around the planet near $6 R_{\mathrm{j}}$. Centrifugal force from Jupiter's rapid rotation drives magnetic flux interchange instabilities ${ }^{11,12}$ whereby outward transport of cold dense plasma is replaced by inward transport of low-density higher-energy $(\sim 1-100 \mathrm{keV})$ plasma. As the plasma is transported into increasing magnetic-field strength, a temperature anisotropy develops whereby the temperature perpendicular to the magnetic field increases faster than that parallel to the field. This anisotropy can excite very low-frequency whistler-mode chorus waves which resonate with electrons ${ }^{13-15}$.

Figure 1 shows data from the Galileo spacecraft where a broad frequency band of whistler-mode chorus waves can be identified below the electron gyrofrequency $\left(f_{\mathrm{ce}}=|e| B /\left(2 \pi m_{\mathrm{e}}\right)\right.$, where $|e|$ is the electron charge, $m_{\mathrm{e}}$ is the electron mass and $B$ is the ambient magnetic-field strength obtained from the fluxgate magnetometer onboard the spacecraft). The band rises and falls in frequency each time the spacecraft crosses the magnetic equator. The waves were detected between 9 and $12 R_{\mathrm{j}}$ in a region where magnetic flux interchange takes place. A more general survey of all Galileo wave data recorded between 27 June 1996 and 5 November 2002 (Fig. 3a) shows that whistler-mode wave intensity (averaged over all longitudes) peaks in a region between 6 and $10 R_{\mathrm{j}}$. Thus magnetic flux interchange beyond the moon Io provides a natural and plentiful source of energy to drive the waves unstable.
To determine whether the waves detected by Galileo interact with relativistic electrons, we calculated the electron resonant energy by solving the cold plasma dispersion relation with the Doppler-shifted cyclotron resonance condition given by

$$
\omega+n\left|\Omega_{\mathrm{e}}\right| / \gamma-k_{\|} v_{\|}=0
$$

where $\gamma$ is the relativistic correction factor, $n$ is the harmonic number $(n=0, \pm 1, \pm 2, \ldots),\left|\Omega_{\mathrm{e}}\right|$ is the cyclotron frequency $\left(=2 \pi f_{\mathrm{ce}}\right)$ and $k_{\|}$and $v_{\|}$are the wavevector and electron velocity component along the ambient magnetic field $\mathbf{B}$. The cyclotron (or gyro-) resonant electron energies for the broad band of waves observed between 13:20 and 13:30 spacecraft event time (SCET) are shown in Fig. 2 for the dominant cyclotron resonance $n=-1$ and wave propagation parallel to $\mathbf{B}$. All electrons in the region between the two black solid curves, which correspond to the upper and lower frequency band of the waves, are diffused in pitch angle and energy by the waves. For a weak temperature anisotropy, lowenergy electrons $(<300 \mathrm{keV})$ are diffused along the red diffusion paths ${ }^{6}$ into the atmospheric loss cone at small pitch angles giving rise to wave growth. For a quasi-isotropic distribution at high energies, diffusion at large pitch angles occurs preferentially in a direction away from the loss cone and resonant electrons gain energy. In effect, the waves transfer energy from a large number of low-energy electrons to accelerate a fraction of the population to high energies. Acceleration occurs via gyro-resonance where the wave frequency is Doppler-shifted to the gyrofrequency of the electrons owing to the relative motion of the waves and particles along the magnetic field. Although the diffusion in Fig. 2 seems small, if the waves persist for a long time ( hours), then the resulting energy diffusion can be very substantial, as it is for the Earth's radiation belts ${ }^{16,17}$. Thus, as resonance can extend from energies of $\sim 10 \mathrm{keV}$ up to several megaelectronvolts, gyro-resonant electron acceleration is possible for plasma conditions at Jupiter.

Wave acceleration is more efficient when the phase velocity of the waves is high, which occurs when the ratio $f_{\mathrm{pe}} / f_{\mathrm{ce}}$ is $\operatorname{low}^{18}$, typically $<4$, where $f_{\mathrm{pe}}$ is the electron plasma frequency given by $f_{\mathrm{pe}}=\left(n_{\mathrm{e}} e^{2} /\left(m_{\mathrm{e}} \varepsilon_{\mathrm{o}}\right)\right)^{1 / 2}, n_{\mathrm{e}}$ is the electron density and $\varepsilon_{\mathrm{o}}$ is the permittivity of free space. Using a plasma density model for Jupiter $^{19}$, and a dipole magnetic field, $f_{\mathrm{pe}} / f_{\mathrm{ce}}$ is high within the Io torus (Fig. $3 \mathrm{~b}$ ), but falls to a minimum between 10 and $20 R_{\mathrm{j}}$ owing to the rapid drop in plasma density. Furthermore, plasma is confined to a thin disc near the centrifugal equator so that $f_{\mathrm{pe}} / f_{\mathrm{ce}}$ 


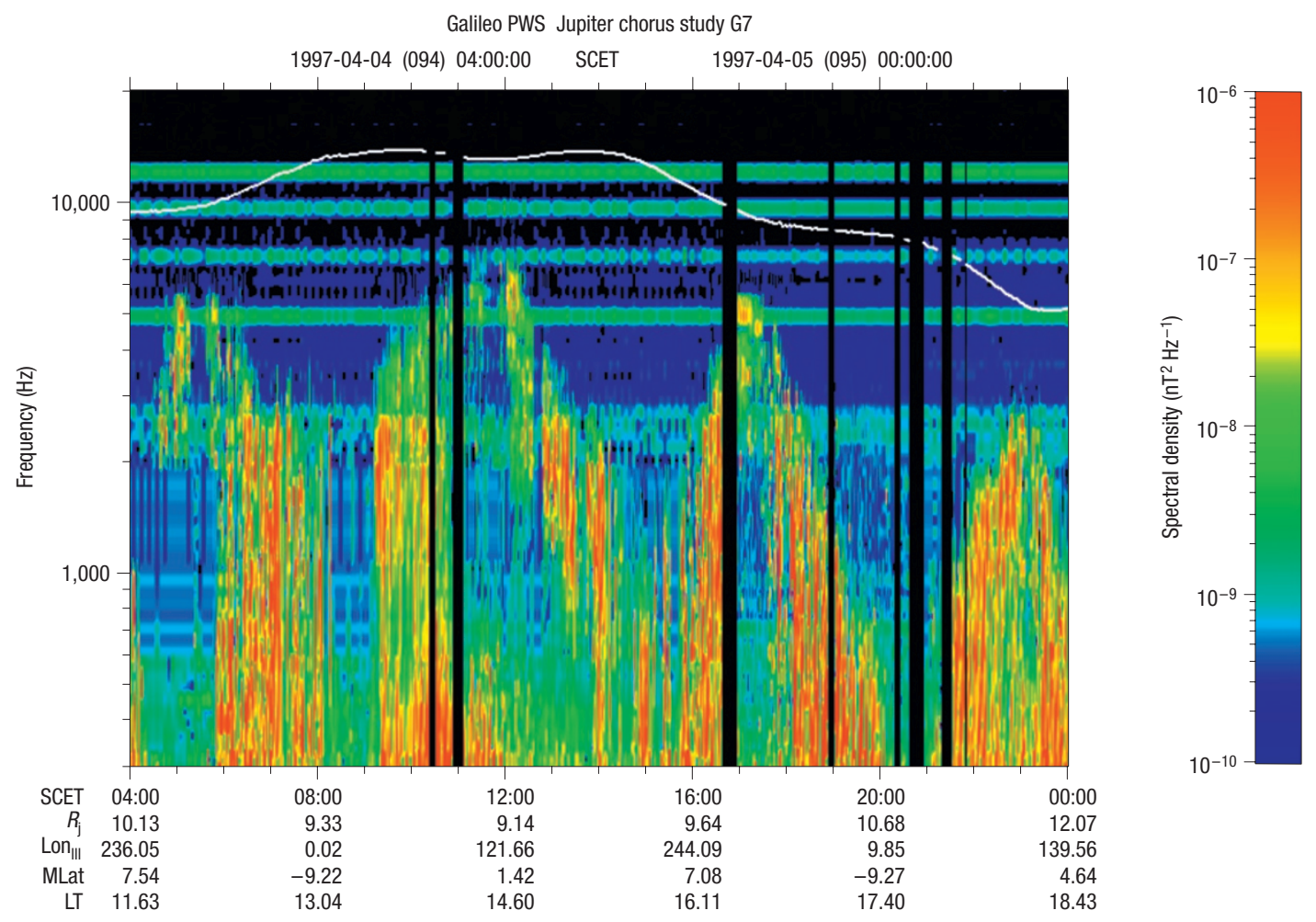

Figure 1 Plasma wave emissions detected by the Galileo plasma wave science (PWS) instrument. The power spectral density of the wave magnetic-field component of electromagnetic waves is shown by the colour bar (in $\mathrm{nT}^{2} \mathrm{~Hz}^{-1}$ ) for SCET, distance from the planet $\left(R_{\mathrm{j}}\right)$, System 3 jovian longitude (Lon ${ }_{I I}$ ), magnetic latitude (MLat) and local time (LT). The local electron gyrofrequency $f_{c e}$ is denoted by the white line. The band of wave power falling in frequency between 17:00 and 20:00 SCET is identified as whistler-mode chorus waves. The frequency of the chorus band increases every time the satellite crosses the magnetic equator with similar rising and falling frequency bands on approach and departure. Note that the waves remain strong as the satellite moves to higher latitudes but are either absent or below the lower frequency of the instrument for latitudes $<-9^{\circ}$.

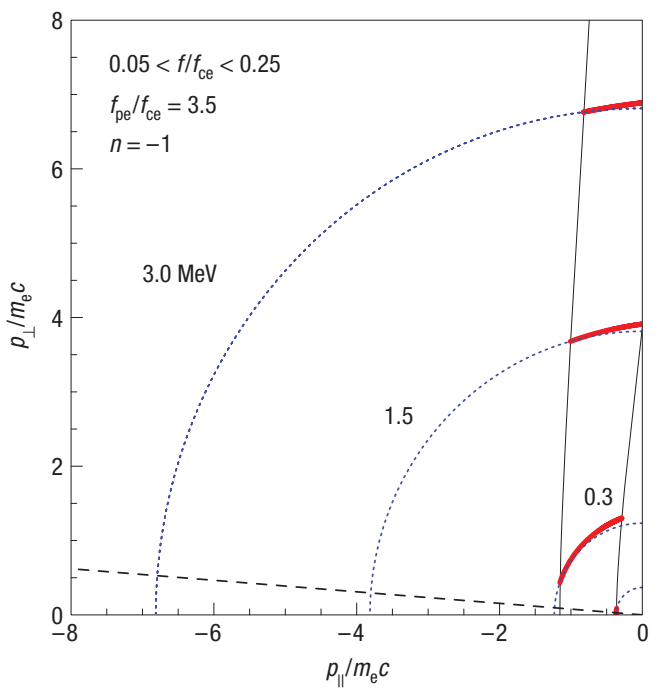

Figure 2 Electron resonant diffusion. Electron resonant energies corresponding to the band of whistler-mode waves observed between 13:20 and 13:30 SCET (Fig. 1) near the magnetic equator at $10 R_{\mathrm{j}}$ are shown by the solid black lines for a typical ratio of the electron plasma frequency to gyrofrequency $\left(f_{\mathrm{pe}} / f_{\mathrm{ce}}=3.5\right)$. Electron resonant diffusion occurs in the region between the solid black lines. Selected diffusion paths are shown in red and constant energy surfaces in blue. The dashed line indicates the atmospheric loss cone. decreases with increasing magnetic latitude, $f_{\mathrm{pe}} / f_{\mathrm{ce}} \sim 2.5$ at $15^{\circ}$ (not shown). Thus the region outside the orbit of Io provides the best conditions for gyro-resonant wave acceleration and suggests that there could be an important contribution from waves at higher latitudes which Galileo did not sample.

To determine whether wave acceleration is effective, we use quasilinear theory to calculate the rate of electron energy diffusion caused by the waves. This approach assumes a broad band of waves with random phase that cause stochastic scattering of the particles. Particle trapping and nonlinear effects are omitted. We calculated pitch angle and energy diffusion rates with the PADIE $\operatorname{code}^{20}$, which assumes that the wave power is gaussian in frequency. In Fig. 1, chorus tends to be strong $\left(2.5 \times 10^{-6} \mathrm{nT}^{2} \mathrm{~Hz}^{-1}\right)$ near $\sim 0.1-0.2 f_{\text {ce }}$ (for example, between 13:20 and 13:30 SCET), and so this was used to define the basic model with $f_{\max }=0.15 f_{\text {ce }}$ at the magnetic equator and a width of $0.05 f_{\text {ce }}$. Although wave power tends to decrease in frequency with increasing latitude (which may indicate non-field aligned propagation), and whistler-mode hiss may be present near a few hundred hertz, $f_{\max }$ is constant in our model. As a result, wave power may be omitted at lower frequencies so that diffusion rates $>10 \mathrm{MeV}$ are probably underestimated.

The direction of wave propagation, or wave normal angle $\psi$, cannot be determined by Galileo as it does not measure all of the wave-field components. However, as whistler-mode chorus waves tend to propagate along $\mathbf{B}$, we assumed a gaussian distribution of wave normal angles in $X=\tan \psi$, centred about the direction of $\mathbf{B}$ with a width $X_{\mathrm{w}}=\tan 30^{\circ}$. This is similar to that used for chorus studies at the Earth ${ }^{16-18}$. For wave 
a

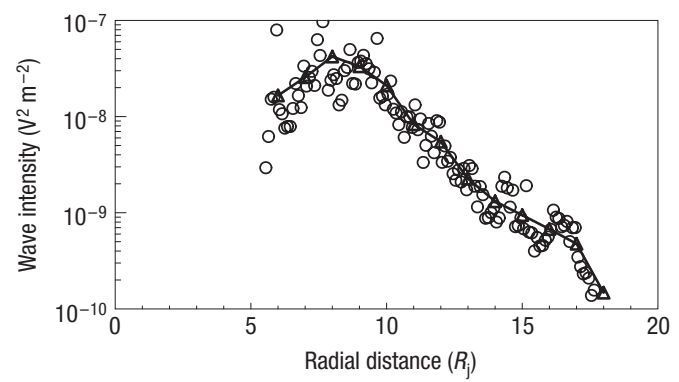

b

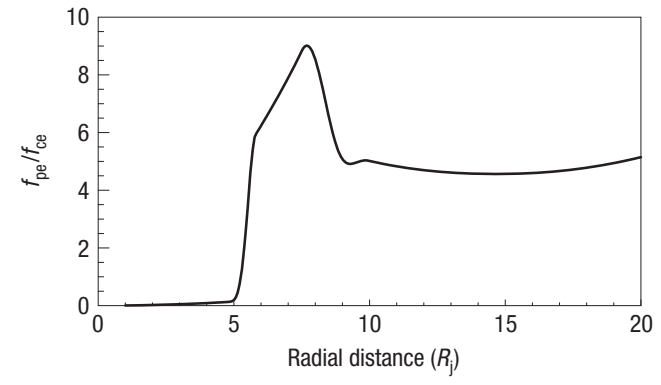

G

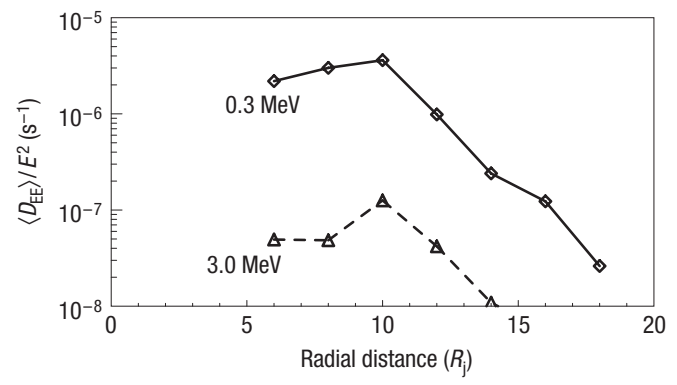

Figure 3 Effects of wave-power, plasma-density and magnetic-field variations on electron energy diffusion. a, Whistler-mode wave intensities measured by Galileo (averaged over all longitudes) within $10^{\circ}$ of the magnetic equator. The wave intensity was obtained by integrating the wave electric-field power spectral density over the frequency band of chorus waves. b, Ratio $f_{\mathrm{pe}} / f_{\mathrm{ce}}$ at the magnetic equator. c, Bounce-averaged energy diffusion coefficients averaged over pitch angles of $70^{\circ}-90^{\circ}$.

propagation at an angle to $\mathbf{B}$, electron diffusion by Landau $(n=0$ in equation (1)) and higher-order $(|n|>1)$ cyclotron harmonic resonances becomes important, although the contribution from higher harmonics becomes progressively weaker. Therefore, the $n=0$ and $n= \pm 1, \pm 2, \ldots, \pm 5$ cyclotron harmonic resonances, which were found to be sufficient, were included in the calculations. As electrons outside the loss cone 'bounce' between mirror points at higher magnetic latitudes, resonant interactions were included (bounce-averaged) for all latitudes within $10^{\circ}$ of the magnetic equator corresponding to the region where strong waves were detected by Galileo. Variations in the plasma density and dipole magnetic field were included in the bounce-averaging. Finally, the wave power at $10 R_{\mathrm{j}}$ was scaled according to the average wave intensity (Fig. 3a) to calculate the diffusion rates at each $R_{\mathrm{j}}$.

Figure $3 c$ shows the bounce-averaged energy diffusion rates averaged between pitch angles of $70^{\circ}$ and $90^{\circ}$. Electron energy diffusion is most effective near $10 R_{\mathrm{j}}$, in the region where $f_{\mathrm{pe}} / f_{\mathrm{ce}}$ falls to a minimum and wave intensity remains high. Energy diffusion is most efficient at energies of a few hundred kiloelectronvolts, but extends up to several megaelectronvolts, and can therefore provide an effective source of electron acceleration.
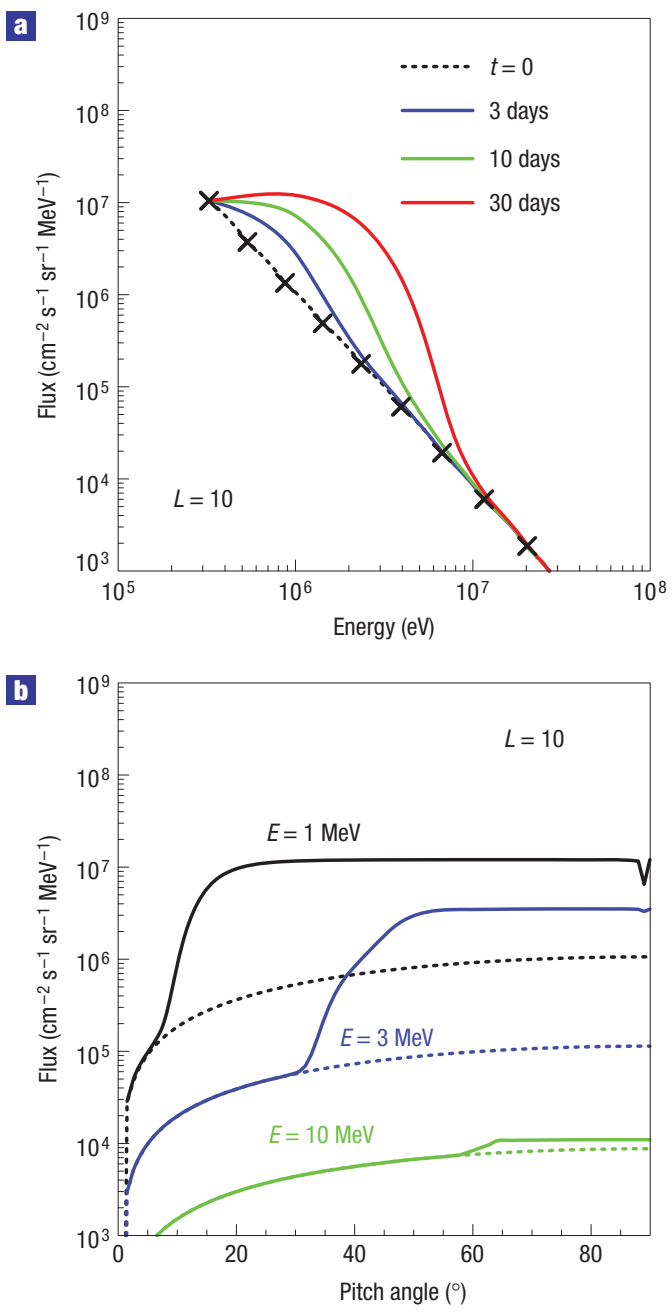

Figure 4 Simulation results. a, Increase in electron flux due to gyro-resonant acceleration by whistler-mode chorus waves. $\mathbf{b}$, Change in pitch angle distribution at selected energies. The dotted lines show the initial conditions obtained from the measured average electron flux.

To calculate the increase in electron flux and the timescale for electron acceleration, we solved a two-dimensional Fokker-Planck equation (given by equation (2) of ref. 17) at $10 R_{\mathrm{j}}$ where acceleration should be most effective. For simplicity, the mixed pitch angle-energy diffusion terms were omitted and so our results should underestimate energy diffusion at large pitch angles ${ }^{21}$. To avoid using data from $10 R_{\mathrm{j}}$ as an initial condition, which may contain contributions from wave acceleration, the average electron flux from Pioneer and Voyager data at $15.75 R_{\mathrm{j}}$ (refs 22,23) was taken as the initial value and mapped to $10 R_{\dot{j}}$ assuming a dipole magnetic field and loss-free inward radial diffusion. The flux was interpolated onto a high-resolution grid, and converted into phase-space density. Bounce-averaged pitch angle and energy diffusion rates from PADIE were used with a pitch angle resolution better than $1^{\circ}$. The average measured electron flux was used as a fixed lower boundary at $300 \mathrm{keV}$ where energy diffusion should exceed losses to the atmosphere. The flux was set to zero at $100 \mathrm{MeV}$, zero inside the loss cone and a flat gradient at $90^{\circ}$. Electrons scattered into the loss cone were assumed to be lost within a quarter of the bounce time. 


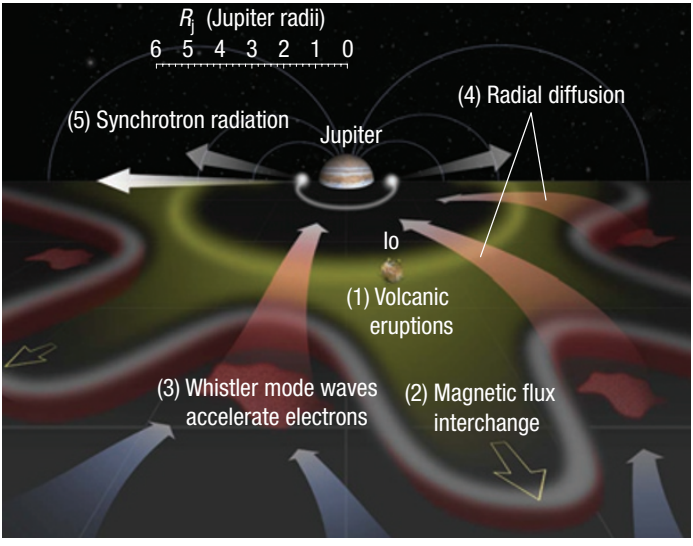

Figure 5 Production of synchrotron radiation from Jupiter. (1) Volcanic gases from lo are ionized and form a cold dense plasma torus around Jupiter. (2) Jupiter's rapid rotation drives magnetic flux interchange and excites whistler-mode waves. (3) Gyro-resonant wave-particle interactions accelerate electrons to relativistic (megaelectronvolt) energies. (4) Radial diffusion transports electrons towards the planet and accelerates them to even higher energies via betatron and Fermi processes. (5) Intense synchrotron radiation is emitted from ultra-relativistic electrons close to the planet $\left(1.4 R_{\mathrm{j}}\right)$.

In the absence of radial transport, the electron flux between 1 and $6 \mathrm{MeV}$ increases by more than an order of magnitude after a period of 30 days (Fig. $4 \mathrm{a}$ ), which is comparable to the timescale for transport (20-50 days) near the Io torus for thermal plasma ${ }^{24}$. The timescale and energy range suggest that gyro-resonant wave acceleration is important outside the orbit of Io. In reality, the peak flux would be reduced by radial diffusion until quasi-equilibrium is obtained.

The model also predicts that the pitch angle distribution between 6 and $15 R_{\mathrm{j}}$ should become peaked near $90^{\circ}$ with a 'flat top' (Fig. 4b), and that the width of the 'flat top' becomes narrower with increasing energy. Electron distributions peaked near $90^{\circ}$ have been reported ${ }^{25}$ near $9 R_{\mathrm{j}}$ for $E>0.55 \mathrm{MeV}$, but have been ascribed to inward radial diffusion. Here, we show they are a signature of wave acceleration.

As electrons are accelerated by gyro-resonant interactions, radial diffusion will transport them inwards towards the planet. As a result, the electron distribution should become even more anisotropic in the region of intense synchrotron radiation $\left(\sim 1.4 R_{\mathrm{j}}\right)$ owing to the radial diffusion process itself, and local losses, particularly the sweeping effect of the jovian moons ${ }^{3}$. Furthermore, whereas most theories suggest that inward radial transport is important in the middle magnetosphere $\left(10-50 R_{\mathrm{j}}\right)$, if gyroresonant wave acceleration between 6 and $15 R_{\mathrm{j}}$ is sufficiently strong to create a peak in electron phase-space density (proportional to measured flux divided by the square of the electron momentum), then radial diffusion will transport the higher-energy electrons outwards as well as inwards to remove the gradient.

The model is sensitive to a number of factors. If the wave spectrum were centred at a lower frequency, it would increase energy diffusion at higher energies and reduce it at lower energies. In addition, wave power at higher latitudes, which has been omitted, may contribute significantly to acceleration because $f_{\mathrm{pe}} / f_{\mathrm{ce}}$ drops rapidly. Thus, wave acceleration could be effective at higher energies. Conversely, if the wave normal angles are larger than $\sim 50^{\circ}$, then the diffusion rates may be much smaller. However, large wave normal angles are unlikely close to the magnetic equator where wave growth is strongest for field-aligned propagation.

We suggest that gyro-resonant electron acceleration is an important part of a multi-step process to produce Jupiter's synchrotron radiation, as presented in Fig. 5. (1) Volcanic eruptions on the moon Io provide a source of particles which are ionized and form a torus around Jupiter. (2) Jupiter's rapid rotation creates magnetic flux interchange instabilities resulting in outward transport of cold dense plasma and inward transport of $\sim 1-100 \mathrm{keV}$ plasma which develops a temperature anisotropy and excites whistler-mode chorus waves. (3) Gyro-resonant electron acceleration accelerates electrons up to several megaelectronvolts outside the orbit of Io. (4) Radial diffusion transports electrons towards the planet ${ }^{3,4}$ and accelerates them up to ultra-relativistic $(\sim 50 \mathrm{MeV})$ energies via betatron and Fermi processes. (5) Only electrons transported close to the planet emit intense synchrotron radiation because the wave power scales at $B^{2} W^{2}$, where $W$ is the electron energy.

Received 22 October 2007; accepted 31 January 2008; published 9 March 2008.

\section{References}

1. Bolton, S. J. et al. Ultra-relativistic electrons in Jupiter's radiation belts. Nature 415, 987-991 (2002)

2. Brice, N. \& McDonough, T. R. Jupiter's radiation belts. Icarus 18, 206-219 (1973).

3. Santo-Costa, D. \& Bourdarie, S. A. Modeling the inner jovian electron radiation belt including non-equatorial particles. Planet. Space Sci. 49, 303-312 (2001).

4. Sicard, A. \& Bourdarie, S. Physical electron belt model from Jupiter's surface to the orbit of Europa. J. Geophys. Res. 109, A02216 (2004).

5. Horne, R. B. \& Thorne, R. M. Potential waves for relativistic electron scattering and stochastic acceleration during magnetic storms. Geophys. Res. Lett. 25, 3011-3014 (1998).

6. Summers, D., Thorne, R. M. \& Xiao, F. Relativistic theory of wave-particle resonant diffusion with application to electron acceleration in the magnetosphere. J. Geophys. Res. 103, 20487-20500 (1998).

Chen, Y., Reeves, G. D. \& Friedel, R. H. W. The energization of relativistic electrons in the outer Van Allen radiation belt. Nature Phys. 3, 614-617 (2007).

8. Horne, R. B. Acceleration of killer electrons. Nature Phys. 3, 590-591 (2007).

9. Sloanaker, R. M. Apparent temperature of Jupiter at a wave length of $10 \mathrm{~cm}$. Astron. J. 64, 346-364 (1959).

10. Hill, T. W., Dessler, A. J. \& Goertz, C. K. in Physics of the Jovian Magnetosphere (ed. Dessler, A. J.) 353 (Cambridge Univ. Press, New York, 1983).

11. Siscoe, G. L. \& Summers, D. Centrifugally driven diffusion of Iogenic plasma. J. Geophys. Res. 86 $8471-8479$ (1981).

12. Kivelson, M. G., Khurana, K. K., Russell, C. T. \& Walker, R. J. Intermittent short-duration plasma-field anomalies in the Io plasma torus: Evidence for interchange in the Io plasma torus? Geophys. Res. Lett. 24, 2127-2130 (1997).

13. Bolton, S. J., Thorne, R. M., Gurnett, D. A., Kurth, W. S. \& Williams, D. J. Enhanced whistler-mode emissions: Signatures of interchange motion in the Io torus. Geophys. Res. Lett. 24, 2123-2126 (1997).

14. Thorne, R. M. et al. Galileo evidence for rapid interchange transport in the Io torus. Geophys. Res. Lett. 24, 2131-2134 (1997)

15. Xiao, F., Thorne, R. M., Gurnett, D. A. \& Williams, D. J. Whistler-mode excitation and electron scattering during an interchange event near Io. Geophys. Res. Lett. 30, 1749 (2003).

16. Horne, R. B. et al. Wave acceleration of electrons in the Van Allen radiation belts. Nature 437, 227-230 (2005).

17. Shprits, Y. Y. et al. Acceleration mechanism responsible for the formation of the new radiation belt during the 2003 Halloween Solar storm. Geophys. Res. Lett. 33, L05104 (2006).

18. Horne, R. B., Glauert, S. A. \& Thorne, R. M. Resonant diffusion of radiation belt electrons by whistler-mode chorus. Geophys. Res. Lett. 30, 1493 (2003).

19. Bagenal, F. Empirical model of the Io plasma torus: Voyager measurements. J. Geophys. Res. 99, 11043-11062 (1994)

20. Glauert, S. A. \& Horne, R. B. Calculation of pitch angle \& energy diffusion coefficients with the PADIE code. J. Geophys. Res. 110, A04206 (2005).

21. Albert, J. M. \& Young, S. L. Multidimensional quasi-linear diffusion of radiation belt electrons. Geophys. Res. Lett. 32, L14110 (2005).

22. Divine, N. \& Garrett, H. B. Charged particle distributions in Jupiter's magnetosphere. J. Geophys. Res. 88, 6889-6903 (1983).

23. Garrett, H. B. et al. Galileo Interim Radiation Electron Model, JPL publication 03-006 72 (The Jet Propulsion Laboratory, Pasadena, 2003).

24. Delamere, P. A. \& Bagenal, F. Modelling variability of plasma conditions in the Io torus. J. Geophys. Res. 108, 1276 (2003)

25. Sentman, D. D. \& Van Allen, J. A. Angular distributions of electrons of energy $E>0.06 \mathrm{MeV}$ in the jovian magnetosphere. J. Geophys. Res. 81, 1350-1360 (1976).

\section{Acknowledgements}

We thank H. B. Garrett and B. Mauk for providing electron data, F. Bagenal for her plasma density model and J. Oliver for producing Fig. 5. This work was supported by the UK NERC, NSF grants ATM-0402615 and ATM-0603191 and NASA grant NNM06AA75C

Correspondence and requests for materials should be addressed to R.B.H.

Author contributions

R.B.H. and R.M.T. led the concept, theory and modelling, S.A.G. and Y.Y.S. contributed modelling, and J.D.M. and D.A.G. contributed data analysis and interpretation.

Reprints and permission information is available online at http://npg.nature.com/reprintsandpermissions/ 